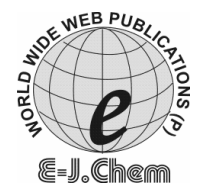

ISSN: 0973-4945; CODEN ECJHAO

E-Journal of Chemistry

http://www.e-journals.net

2010, 7(1), 55-58

\title{
Synthesis of Spiro Heterocyclic Compounds
}

\author{
MOHAMED N IBRAHIM*, MOHAMED F EL-MESSMARY \\ and MOHAMED G A ELARFI
}
Chemistry Department, Faculty of Science, Garyounis University, Benghazi, Libya.
mnibrahim46@yahoo.com

Received 3 April 2009; Accepted 14 May 2009

\begin{abstract}
Reaction of isatin with acetophenone derivatives gave 3-hydroxy-3phenacyl oxindole derivatives (II), dehydration of (II) gave 3-phenacylidene-2indolinone derivatives (III). Condensation of (III) with hydrazine hydrate, phenylhydrazine and phenylthiourea afforded new spiropyrazolines (IV \& V) and spiropyrimidinethione (VI) respectively. The structures of the final products were established by physical and spectral means.
\end{abstract}

Keywords: Synthesis, Isatin, Spiropyrazolines, Spiropyrimidinethione.

\section{Introduction}

The reaction of isatin with various compounds have been under intensive studies by many authors $^{1-6}$ and due to the importance of some derivatives, for example, pyrazoline and pyrimidinethione in different biological and industrial aspects ${ }^{7,8}$, this research work has been initiated to prepare such new molecules. The reaction of isatin with acetophenone derivatives ( $\left.\mathbf{I}_{\text {a-c }}\right)$ was carried out in the presence of diethyl amine as a basic catalyst giving rise to 3-hydroxy-3-phenacyl oxindoles $\left(\mathbf{I}_{\mathbf{a - c}}\right)$ in good yields. Dehydration of the above compounds by dilute alcoholic hydrochloric acid gave 3-phenacylidene-2-indolinones ( III $_{\text {a-c }}$ ) in quantitative yields. Cyclocondensation of $\alpha, \beta$-unsaturated ketones with hydrazine have been previously investigated ${ }^{9,10}$ in which most cases gave pyrazoline. Accordingly the reaction of compound (III) with hydrazine, phenylhydrazine were carried out in presence of diethyl amine and gave spiropyrazoline (IV) and spiro- $N$-phenylpyrazoline (V) respectively. On the other hand interaction of (III) with phenylthiourea in the presence of alcoholic $\mathrm{KOH}^{11}$ afforded spiropyrimidinethione (VI), (Scheme 1). The structures of the final products were established by physical and spectral methods (Table 1).

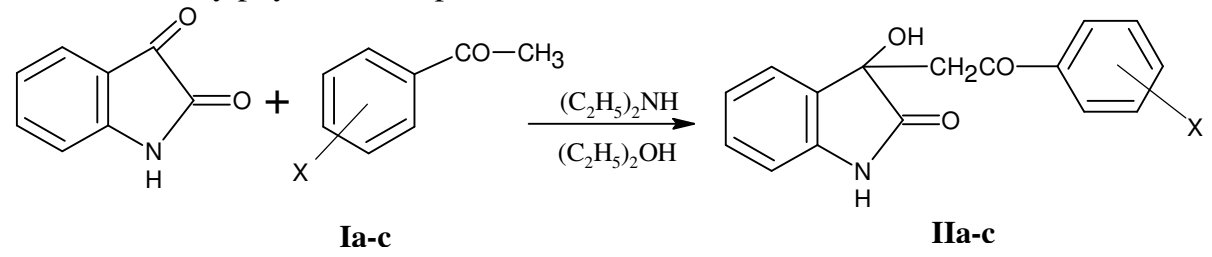




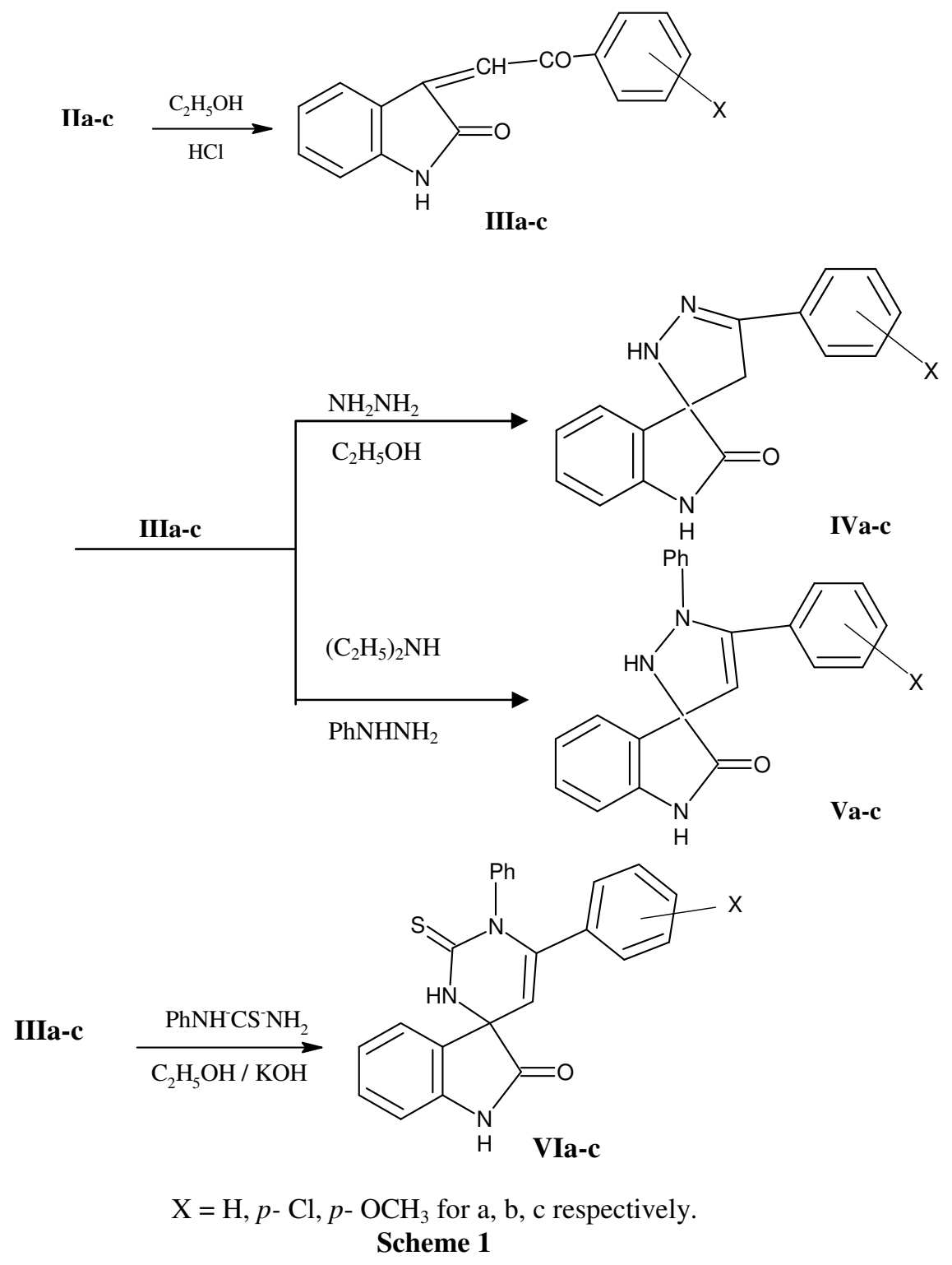

\section{Experimental}

Melting points were measured on Gallen-Kamp apparatus and are uncorrected. I.R. spectra were recorded on a Unicam SP1100 spectrophotometer. ${ }^{1} \mathrm{H}-\mathrm{NMR}$ spectra were recorded in $\mathrm{CDCl}_{3}$ on a Varian Mercury VX spectrometer at $300 \mathrm{MHz}$, using TMS as internal reference.

Preparation of 3-hydroxy-3-phenacyl oxindoles ( $\left.\boldsymbol{I I}_{\text {a-c }}\right)$

A mixture of isatin and substituted acetophenone ( 0.01 mole of each) was dissolved in ethanol $(100 \mathrm{~mL})$ and diethyl amine $(1 \mathrm{~mL})$ was added. The mixture was allowed to stand overnight at room temperature, the yellow needles formed were recrystallised from ethanol. 
Preparation of 3-phenacylidene-2-indolinones $\left(\boldsymbol{I I \boldsymbol { I } _ { \boldsymbol { a } - \mathrm { c } }}\right)$

A mixture of 0.01 mole of compound $\left(\mathbf{I I}_{\mathrm{a}-\mathrm{c}}\right)$, ethanol $25 \mathrm{~mL}$ and $50 \mathrm{~mL}$ of dilute $\mathrm{HCl}$ solution $(25 \%)$, was allowed to stand overnight, fine orange needles were formed.

Preparation of spiropyrazolines $\left(\boldsymbol{I} \boldsymbol{V}_{a-c} \& \boldsymbol{V}_{a-c}\right)$

A mixture of 0.01 mole of compound ( III $_{\mathrm{a}-\mathrm{c}}$ ) and hydrazine hydrate $(50 \%)$ or phenyl hydrazine $(0.01 \mathrm{~mole})$ in ethanol $(50 \mathrm{~mL})$ and diethyl amine $(1 \mathrm{~mL})$ was refluxed for $10-14$ hours, then acetic acid $(10 \mathrm{ml})$ was added to the cold solution. The precipitate formed after concentration was filtered and washed with cold ethanol and recrystallised from acetic acid.

Preparation of spiropyrimidinethione ( $\boldsymbol{V I}_{\text {a-c }}$ )

A mixture of 0.01 mole of (III a-c $)$, phenylthiourea (0.02 mol), $\mathrm{KOH}(2 \mathrm{~g})$, ethanol $(60 \mathrm{~mL})$ and water $(10 \mathrm{~mL})$ was refluxed for $10-14$ hours. The heavy precipitate formed after concentration and cooling was filtered, dried and recrystallised from ethanol.

Table 1. Physical and spectral data of compounds ( $\left.\mathbf{I I}_{\mathrm{a}-\mathrm{c}}-\mathbf{V \mathbf { I } _ { \mathrm { a } - \mathrm { c } }}\right)$

\begin{tabular}{|c|c|c|c|c|}
\hline $\begin{array}{c}\text { Compd. } \\
\text { No }\end{array}$ & $\begin{array}{c}\mathrm{M} . \mathrm{p}, \\
{ }^{\circ} \mathrm{C}\end{array}$ & $\begin{array}{c}\text { Yield, } \\
\%\end{array}$ & $\begin{array}{c}\text { IR, } \\
\text { v / } \mathrm{Cm}^{-1}\end{array}$ & $\begin{array}{c}{ }^{1} \mathrm{H} \text { NMR, } \\
\delta / \mathrm{ppm}\end{array}$ \\
\hline \multirow[t]{2}{*}{$\mathbf{I I}_{\mathbf{a}}$} & $127-9$ & 65 & $1630(\mathrm{CO}) ; 1705(\mathrm{CO})$ & $3.7\left(\mathrm{~s}, 2 \mathrm{H}, \mathrm{CH}_{2}\right) ; 6.0(\mathrm{~b}, 1 \mathrm{H}, \mathrm{OH})$ \\
\hline & & & $3410(\mathrm{NH}) ; 3500(\mathrm{OH})$ & 7.6(m,9H,Ar-H $) ; 10.1(\mathrm{~s}, 1 \mathrm{H}, \mathrm{NH})$ \\
\hline \multirow{2}{*}{$\mathbf{I I}_{\mathbf{b}}$} & $184-6$ & 76 & $1640(\mathrm{CO}) ; 1685(\mathrm{CO})$ & $3.5\left(\mathrm{~s}, 2 \mathrm{H}, \mathrm{CH}_{2}\right) ; 5.7(\mathrm{~s}, 1 \mathrm{H}, \mathrm{OH})$ \\
\hline & & & $3400(\mathrm{NH}) ; 3440(\mathrm{OH})$ & $7.8(\mathrm{~m}, 8 \mathrm{H}, \mathrm{Ar}-\mathrm{H}) ; 10.2(\mathrm{~s}, 1 \mathrm{H}, \mathrm{NH})$ \\
\hline \multirow[t]{3}{*}{$\mathbf{I I}_{\mathbf{c}}$} & $187-9$ & 72 & $1650(\mathrm{CO}) ; 1710(\mathrm{CO})$ & $3.3\left(\mathrm{~s}, 2 \mathrm{H}, \mathrm{CH}_{2}\right) ; 4.6\left(\mathrm{~s}, 3 \mathrm{H}, \mathrm{OCH}_{3}\right)$ \\
\hline & & & 1100(O-C); 3420(NH) & $5.9(\mathrm{~s}, 1 \mathrm{H}, \mathrm{OH}) ; 7.4(\mathrm{~m}, 8 \mathrm{H}, \mathrm{Ar}-\mathrm{H})$ \\
\hline & & & $3500(\mathrm{OH})$ & $10.0(\mathrm{~s}, 1 \mathrm{H}, \mathrm{NH})$ \\
\hline \multirow[t]{2}{*}{ III $_{\mathbf{a}}$} & $194-6$ & 86 & $1590(\mathrm{C}=\mathrm{C}) ; 1630(\mathrm{CO})$ & 7.6(s,1H,CH $) ; 7.8(\mathrm{~m}, 9 \mathrm{H}, \mathrm{Ar}-\mathrm{H})$ \\
\hline & & & $1690(\mathrm{CO}) ; 3400(\mathrm{NH})$ & $10.8(\mathrm{~s}, 1 \mathrm{H}, \mathrm{NH})$ \\
\hline \multirow[t]{2}{*}{ III $_{b}$} & $182-5$ & 80 & $1580(\mathrm{C}=\mathrm{C}) ; 1620(\mathrm{CO})$ & $7.5(\mathrm{~s}, 1 \mathrm{H}, \mathrm{CH}) ; 7.7(\mathrm{~m}, 8 \mathrm{H}, \mathrm{A}-\mathrm{H})$ \\
\hline & & & $1675(\mathrm{CO}) ; 3400(\mathrm{NH})$ & $10.6(\mathrm{~s}, 1 \mathrm{H}, \mathrm{NH})$ \\
\hline \multirow[t]{2}{*}{ III $_{\mathbf{c}}$} & $192-4$ & 87 & $1580(\mathrm{C}=\mathrm{C}) ; 1640(\mathrm{CO})$ & $4.6\left(\mathrm{~s}, 3 \mathrm{H}, \mathrm{OCH}_{3}\right) ; 7.5(\mathrm{~s}, 1 \mathrm{H}, \mathrm{CH})$ \\
\hline & & & $1690(\mathrm{CO}) ; 3400(\mathrm{NH})$ & $7.8(\mathrm{~m}, 8 \mathrm{H}, \mathrm{Ar}-\mathrm{H}) ; 10.4(\mathrm{~s}, 1 \mathrm{H}, \mathrm{NH})$ \\
\hline \multirow[t]{2}{*}{$\mathbf{I V}_{\mathbf{a}}$} & $262-4$ & 56 & $1240(\mathrm{C}-\mathrm{N}) ; 1625(\mathrm{C}=\mathrm{N})$ & $3.8\left(\mathrm{~s}, 2 \mathrm{H}, \mathrm{CH}_{2}\right) ; 7.7(\mathrm{~m}, 9 \mathrm{H}, \mathrm{Ar}-\mathrm{H})$ \\
\hline & & & $1695(\mathrm{CO}) ; 3300(\mathrm{NH})$ & $10.1(\mathrm{~s}, 2 \mathrm{H}, 2 \mathrm{NH})$ \\
\hline \multirow[t]{2}{*}{$\mathbf{I V}_{\mathrm{b}}$} & $222-3$ & 52 & $1250(\mathrm{C}-\mathrm{N}) ; 1620(\mathrm{C}=\mathrm{N})$ & $3.6\left(\mathrm{~s}, 2 \mathrm{H}, \mathrm{CH}_{2}\right) ; 7.8(\mathrm{~m}, 8 \mathrm{H}, \mathrm{Ar}-\mathrm{H})$ \\
\hline & & & $1690(\mathrm{CO}) ; 3400(\mathrm{NH})$ & $10.1(\mathrm{~s}, 2 \mathrm{H}, 2 \mathrm{NH})$ \\
\hline \multirow[t]{2}{*}{$\mathbf{I} \mathbf{V}_{\mathbf{c}}$} & $226-7$ & 55 & $1240(\mathrm{C}-\mathrm{N}) ; 1630(\mathrm{C}=\mathrm{N})$ & $3.8\left(\mathrm{~s}, 2 \mathrm{H}, \mathrm{CH}_{2}\right) ; 4.4\left(\mathrm{~s}, 3 \mathrm{H}, \mathrm{OCH}_{3}\right)$; \\
\hline & & & $1690(\mathrm{CO}) ; 3340(\mathrm{NH})$ & $7.7(\mathrm{~m}, 8 \mathrm{H}, \mathrm{Ar}-\mathrm{H}) ; 10.2(\mathrm{~s}, 2 \mathrm{H}, 2 \mathrm{NH})$ \\
\hline \multirow[t]{2}{*}{$\mathbf{V}_{\mathbf{a}}$} & $210-2$ & 56 & $1240(\mathrm{C}-\mathrm{N}) ; 1600(\mathrm{C}=\mathrm{C})$ & $6.6(\mathrm{~s}, 1 \mathrm{H}, \mathrm{CH}) ; 8.0(\mathrm{~m}, 14 \mathrm{H}, \mathrm{Ar}-\mathrm{H})$ \\
\hline & & & $1700(\mathrm{CO}) ; 3400(\mathrm{NH})$ & $10.0(\mathrm{~s}, 2 \mathrm{H}, 2 \mathrm{NH})$ \\
\hline \multirow[t]{2}{*}{$\mathbf{V}_{\mathbf{b}}$} & $164-6$ & 60 & $1250(\mathrm{C}-\mathrm{N}) ; 1605(\mathrm{C}=\mathrm{C})$ & $6.7(\mathrm{~s}, 1 \mathrm{H}, \mathrm{CH}) ; 7.9(\mathrm{~m} .13 \mathrm{H}, \mathrm{Ar}-$ \\
\hline & & & $1690(\mathrm{CO}) ; 3390(\mathrm{NH})$ & H) $10.1(\mathrm{~s}, 2 \mathrm{H}, 2 \mathrm{NH})$ \\
\hline \multirow[t]{2}{*}{$\mathbf{V}_{\mathbf{c}}$} & $212-3$ & 55 & $1245(\mathrm{C}-\mathrm{N}) ; 1590(\mathrm{C}=\mathrm{C})$ & $6.5(\mathrm{~s}, 1 \mathrm{H}, \mathrm{CH}) ; 4.4\left(\mathrm{~s}, 3 \mathrm{H}, \mathrm{OCH}_{3}\right)$ \\
\hline & & & 1695(CO); 3400(NH) & $8.0(\mathrm{~m}, 13 \mathrm{H}, \mathrm{Ar}-\mathrm{H}) ; 10.0(\mathrm{~s}, 2 \mathrm{H}, 2 \mathrm{NH})$ \\
\hline \multirow[t]{2}{*}{$\mathbf{V I} \mathbf{I}_{\mathbf{a}}$} & $146-9$ & 60 & $1240(\mathrm{CS}) ; 1605(\mathrm{C}=\mathrm{C})$ & $6.9(\mathrm{~s}, 1 \mathrm{H}, \mathrm{CH}) ; 8.0(\mathrm{~m}, 13 \mathrm{H}, \mathrm{Ar}-\mathrm{H})$ \\
\hline & & & $1695(\mathrm{CO}) ; 3400(\mathrm{NH})$ & $10.0(\mathrm{~s}, 2 \mathrm{H}, 2 \mathrm{NH})$ \\
\hline \multirow[t]{2}{*}{$\mathbf{V I}_{\mathbf{b}}$} & $245-7$ & 57 & $1250(\mathrm{CS}) ; 1580(\mathrm{C}=\mathrm{C})$ & $6.8(\mathrm{~s}, 1 \mathrm{H}, \mathrm{CH}) ; 7.9(\mathrm{~m}, 13 \mathrm{H}, \mathrm{Ar}-\mathrm{H})$ \\
\hline & & & $1695(\mathrm{CO}) ; 3360(\mathrm{NH})$ & $10.1(\mathrm{~s}, 2 \mathrm{H}, 2 \mathrm{NH})$ \\
\hline \multirow[t]{2}{*}{$\mathbf{V I} \mathbf{I}_{\mathbf{c}}$} & $183-5$ & 50 & $1240(\mathrm{CS}) ; 1590(\mathrm{C}=\mathrm{C})$ & $6.6(\mathrm{~s}, 1 \mathrm{H}, \mathrm{CH}) ; 4.6\left(\mathrm{~s}, 3 \mathrm{H}, \mathrm{OCH}_{3}\right)$ \\
\hline & & & $1695(\mathrm{CO}) ; 3400(\mathrm{NH})$ & $8.1(\mathrm{~m}, 13 \mathrm{H}, \mathrm{Ar}-\mathrm{H}) ; 10.1(\mathrm{~s}, 2 \mathrm{H}, 2 \mathrm{NH})$ \\
\hline
\end{tabular}




\section{Results and Discussion}

The first step in this synthesis involve crossed aldol condensation between the acetophenone or its derivatives $\left(\mathbf{I}_{\mathrm{a}-\mathrm{c}}\right)$ with the isatin in basic medium to give the aldol products, 3-hydroxy3-phenacyloxindoles $\left(\mathbf{I}_{\mathbf{a}-\mathrm{c}}\right)$ which then dehydrated easily by using dilute alcoholic hydrochloric acid, to yield the expected $\alpha, \beta$-unsaturated carbonyl compounds, 3phenacylidene-2-indolinones ( III $_{\text {a-c }}$ ). Nucleophilic Michael addition of hydrazine or phenyl hydrazine or phenylthiourea to the above compounds leads to the formation of spiro-5-(-3indolyl-2-one)-3-phenylpyrazoline, spiro-5-(-3-indolyl-2-one)-2, 3-diphenylpyrazolin-3-ene, and spiro-6-(-3-indolyl-2-one)-3, 4-diphenylpyrimidinethione, $\left(\mathbf{I} \mathbf{V}_{\mathrm{a}-\mathrm{c}} ; \mathbf{V}_{\mathrm{a}-\mathrm{c}} ; \mathbf{V I}_{\mathbf{a - c}}\right)$ respectively. The structures of these products were established by physical and spectral methods.

\section{References}

1. Meyers F J H and Lindwall G, J Am Chem Soc., 1938, 60, 466.

2. Noriyasa H, Shigeru S, Shisue K and Sheji T, Chem Pharm Bull., 1973, 21, 910.

3. El-Magraby M A and El-Abbady M, Indian J Chem Soc., 1978, 16B, 57.

4. Shanmugasundram M, Raghunathan R and Malar E J, Heteroatom Chem., 1998, 9, 522.

5. Dawood K M, Tetrahedron, 2005, 61(22), 5229.

6. Dalloul H M, ARKIVOC, 2008, 14, 234.

7. El-Magraby M A, Khalafalla A K, Hassan M E and Soleiman H A, J Indian Chem Soc., 1986, 63, 910.

8. Fahmy A M, Hassan M K, Khalaf A A and Ahmed R A, Revue Roumaine de Chimie, 1988, 33, 755.

9. Smith L A and Howard L L, J Am Chem Soc., 1943, 65, 159

10. Lindwall H G and Mclennan J S, J Am Chem Soc., 1932, 54, 4739.

11. Sammour A, Selim M I B, Nour M and Abd-El-Halim M, J Chem U A R, 1970, 13, 7. 


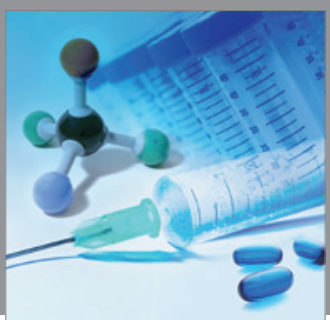

International Journal of

Medicinal Chemistry

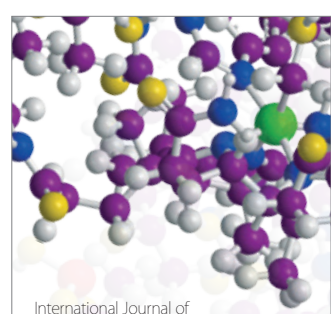

Carbohydrate Chemistry

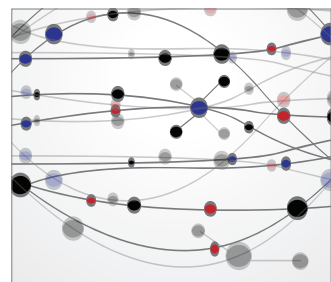

The Scientific World Journal
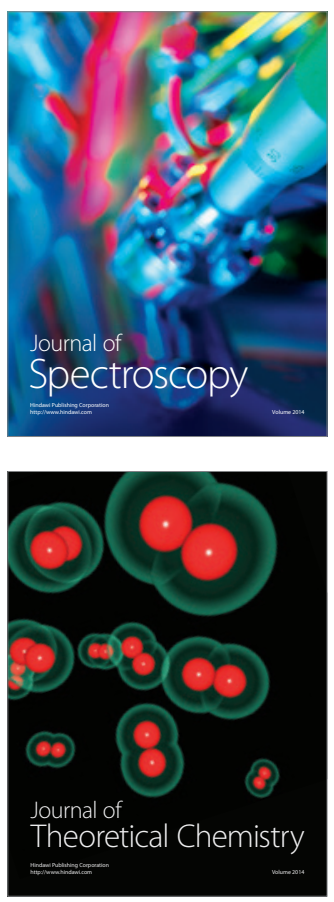
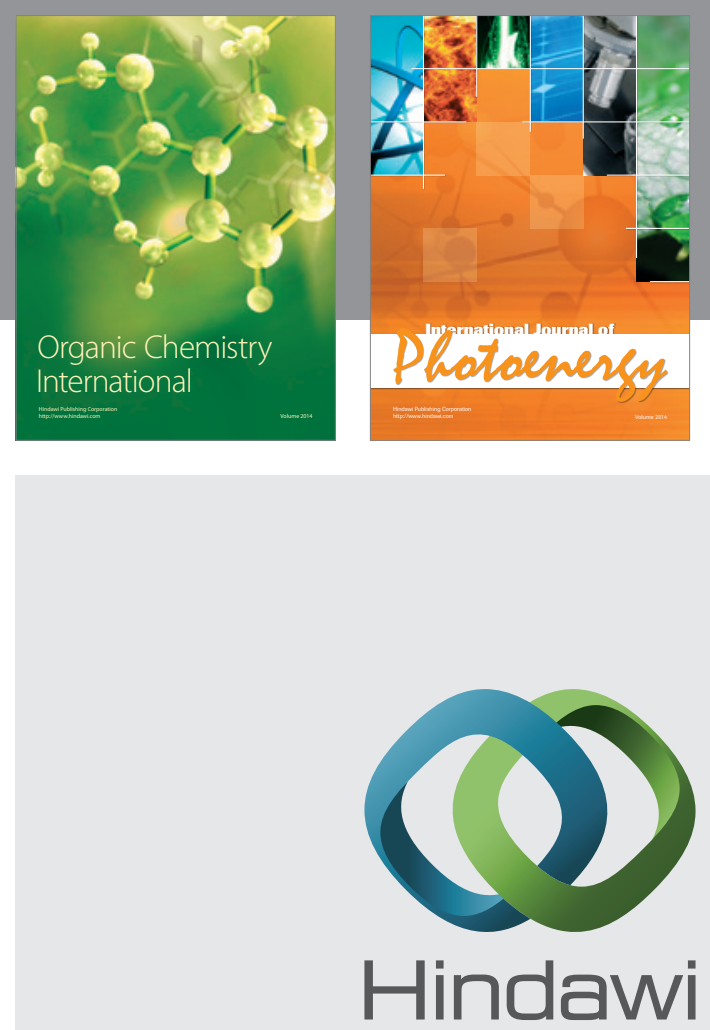

Submit your manuscripts at

http://www.hindawi.com
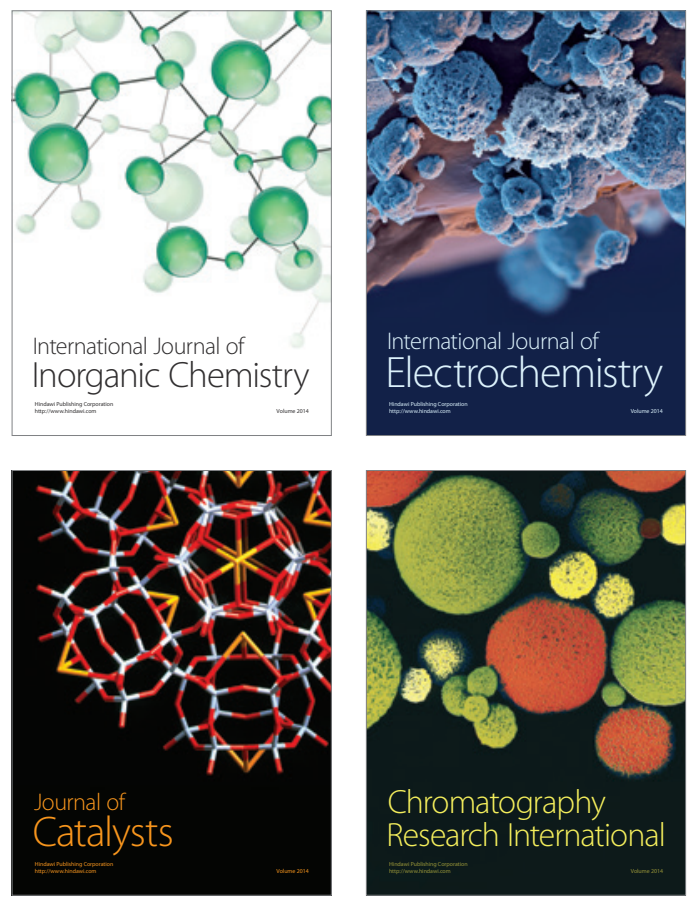
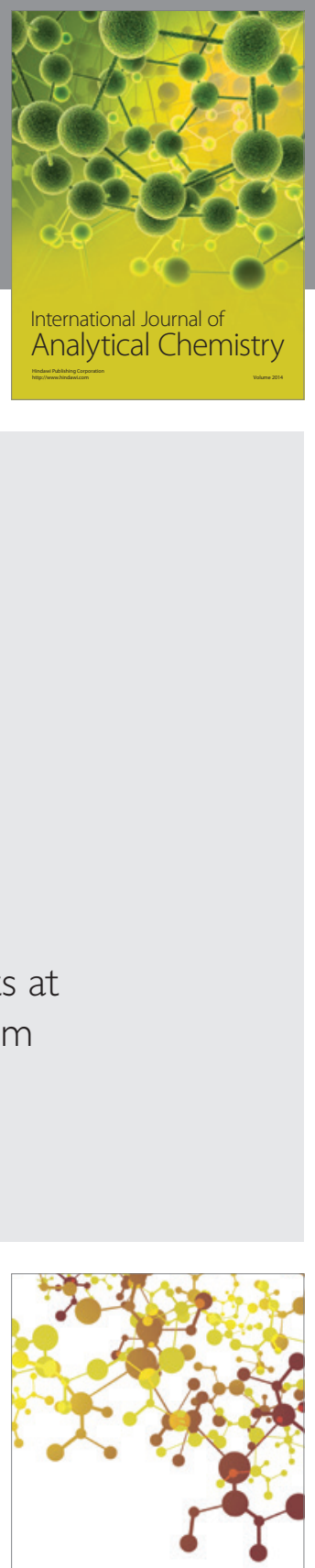

Journal of

Applied Chemistry
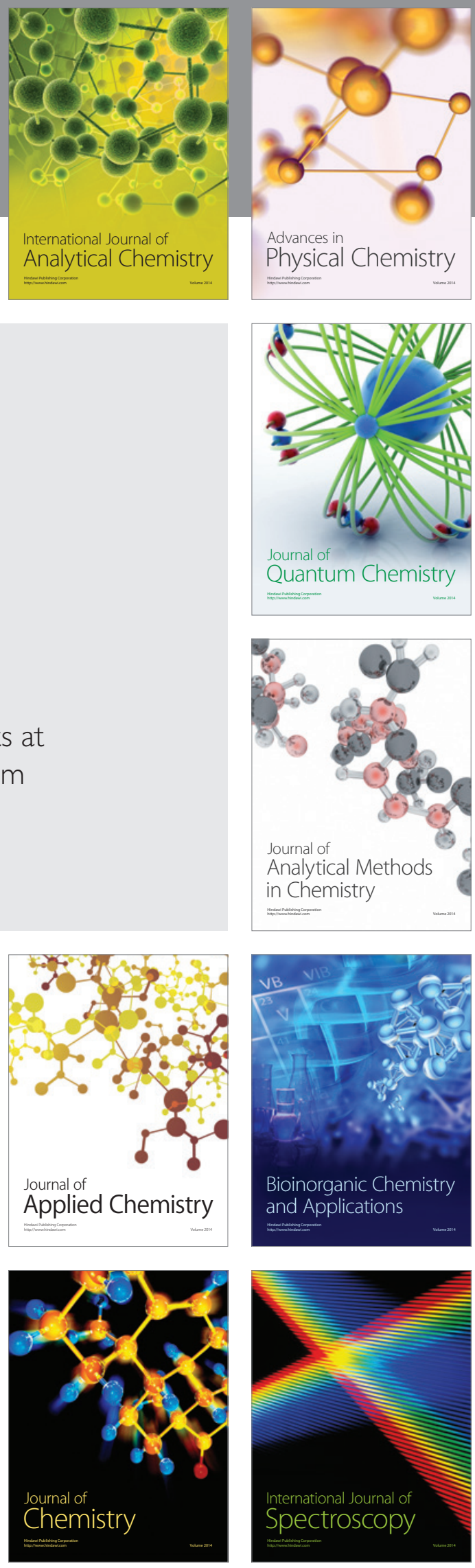